\title{
Ground-based terahertz CO spectroscopy towards Orion
}

\author{
J. Kawamura ${ }^{1, \star}$, T. R. Hunter ${ }^{2}$, C.-Y. E. Tong ${ }^{2}$, R. Blundell ${ }^{2}$, D. C. Papa ${ }^{2}$, F. Patt ${ }^{3}$, W. Peters ${ }^{3}$, T. L. Wilson ${ }^{3,4}$, \\ C. Henkel ${ }^{4}$, G. Gol'tsman ${ }^{5}$, and E. Gershenzon ${ }^{5}$ \\ ${ }^{1}$ California Institute of Technology, Pasadena, California, 91125, USA \\ 2 Harvard-Smithsonian Center for Astrophysics, 60 Garden St., Cambridge, Massachusetts 02138, USA \\ 3 Submillimeter Telescope Observatory, 933 N. Cherry Ave., Tucson, Arizona, 85721, USA \\ 4 Max-Planck Institut für Radioastronomie, Postfach 2024, 53010 Bonn, Germany \\ 5 Moscow State Pedagogical University, Moscow, 119435, Russia
}

Received 9 February 2001 / Accepted 24 July 2002

\begin{abstract}
Using a superconductive hot-electron bolometer heterodyne receiver on the 10-m Heinrich Hertz Telescope on Mount Graham, Arizona, we have obtained velocity-resolved $1.037 \mathrm{THz} \mathrm{CO}(J=9 \rightarrow 8)$ spectra toward several positions along the Orion Molecular Cloud (OMC-1) ridge. We confirm the general results of prior observations of high- $J$ CO lines that show that the high temperature, $T_{\text {kin }} \geq 130 \mathrm{~K}$, high density molecular gas, $n \geq 10^{6} \mathrm{~cm}^{-3}$, is quite extended, found along a $\sim 4^{\prime}$ region centered on BN/KL. However, our observations have significantly improved angular resolution, and with a beam size of $\theta_{\mathrm{FWHP}} \approx 9^{\prime \prime}$ we are able to spatially and kinematically discriminate the emission originating in the extended quiescent ridge from the very strong and broadened emission originating in the compact molecular outflow. The ridge emission very close to the BN/KL region appears to originate from two distinct clouds along the line of sight with $v_{\mathrm{LSR}} \approx+6(1) \mathrm{km} \mathrm{s}^{-1}$ and $\approx+10(1) \mathrm{km} \mathrm{s}^{-1}$. The former component dominates the emission to the south of BN/KL and the latter to the north, with a turnover point coincident with or near BN/KL. Our evidence precludes a simple rotation of the inner ridge and lends support to a model in which there are multiple molecular clouds along the line of sight towards the Orion ridge.
\end{abstract}

Key words. ISM: molecules - ISM: individual objects: Orion KL - submillimeter

\section{Introduction}

At a distance of less than $0.5 \mathrm{kpc}$, the Orion Molecular Cloud (OMC) is the nearest site of massive star formation (Genzel \& Stutzki 1989). The OMC-1 core contains a tight cluster of energetic sources whose properties and relations to one another are difficult to unravel because they are simply so densely packed together. The Becklin-Neugebauer (BN) object, KleinmannLow (KL) infrared nebula, intense $\mathrm{SiO}$ and water vapor masers, and a massive molecular outflow (e.g., Menten \& Reid 1995) all reside in a $\sim 30^{\prime \prime}$-diameter area, which we hereafter refer to as the $\mathrm{BN} / \mathrm{KL}$ region.

When this region is explored with molecular lines, it is possible to separate the emission sources into three components, which are distinguished by their linewidths. In order of increasing velocity dispersion, the three components are the extended quiescent cloud (the ridge), the hot core, and the outflowing and shocked gas. For example, in single-dish observations of low$J$ CO transitions the spectra consist of a blend of emission from the quiescent gas and the outflowing gas. Interferometric observations allow one to resolve the spatially compact outflow, but

Send offprint requests to: J. Kawamura,

e-mail: jonathan.h.kawamura@jpl.nasa.gov

* Present address: Jet Propulsion Laboratory, MS 168-314, California Institute of Technology, Pasadena, California, 91109, USA. filter out much of the emission from the extended ridge. The velocity of the quiescent gas seems to follow a shallow gradient along the ridge, becoming more positive in $v_{\text {LSR }}$ from $\mathrm{N}$ to $\mathrm{S}$. This has been taken as evidence for rotation of the Orion molecular cloud along an axis roughly lying in a E-W direction (Kutner et al. 1976, 1977; Hasegawa et al. 1984; Vogel et al. 1985). Although there may indeed be large-scale rotation of the cloud, more recent observations have shown that the velocity and structure of the OMC ridge is too complicated to be consistent with a simple rotation of the compact ridge (Wilson et al. 1986). Observing these lines using a large ground-based submillimeter telescope offers us an opportunity to study the warm and dense regions directly associated with the energetic sources with good spatial detail. In this paper we present resolved $1.037 \mathrm{THz} \mathrm{CO}(J=9 \rightarrow 8)$ spectra obtained from the ground.

\section{Observations}

Our spectra were taken at the Heinrich Hertz Telescope atop Mt. Graham, Arizona (Baars et al. 1999) using a superconductive hot-electron bolometer (HEB) mixer receiver (Kawamura et al. 2000; Tong et al. 1999). The receiver was tuned to position the $\operatorname{CO}(J=9 \rightarrow 8)$ line, which has a rest frequency of $v=1.0369124 \mathrm{THz}$, in the lower sideband. 


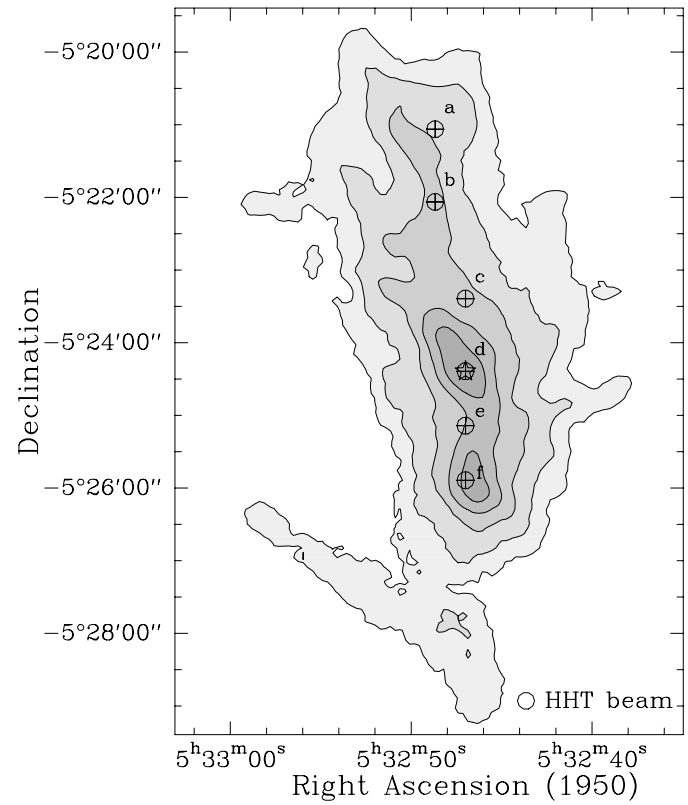

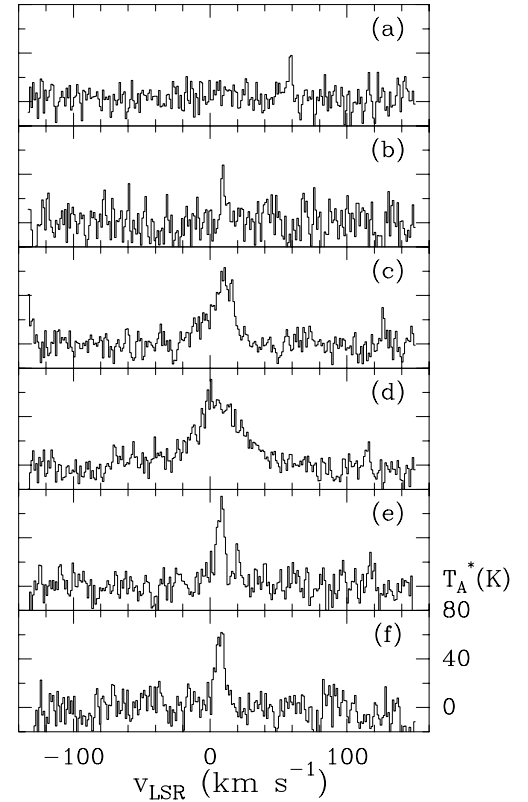

Fig. 1. Terahertz $\mathrm{CO}(J=9 \rightarrow 8)$ spectra taken in Orion. The left panel shows the positions where spectra were taken, indicated by circled crosses. These are marked on a grey scale/contour map of the $350 \mu \mathrm{m}$ continuum dust emission of the region (Lis et al. 1998). The star indicates the position of $\mathrm{BN} / \mathrm{KL}$. The beam size of the HHT observing at $1.037 \mathrm{THz}$ is shown in the lower-right hand corner of the panel. Spectra taken at respective positions are plotted in the panel to the right. The spectra have been smoothed to a velocity resolution of $1.1 \mathrm{~km} \mathrm{~s}^{-1}$.
The double-sideband receiver noise temperature is $T_{\mathrm{RX}}=$ $1600 \mathrm{~K}$. The observations occurred on 7 January 2000, when the $225 \mathrm{GHz}$ sky opacity at zenith, as measured by a tipping radiometer, fell to less than $\tau_{225}<0.04$. Opacity measurements with a $345 \mathrm{GHz}$ SIS receiver made before and after the $\mathrm{THz}$ observations indicated that the upper limit to the precipitable water vapor was between $0.5 \mathrm{~mm}$ and $0.7 \mathrm{~mm}$. The sources were observed using a chopped secondary, for which the throw was $4^{\prime}$ in azimuth and the chop frequency was $1 \mathrm{~Hz}$. The spectra were calibrated using the standard ambient temperature chopper method to obtain spectra calibrated to above the atmosphere, $T_{\mathrm{A}}^{*}$. For sources at high elevation angle, we measured a single-sideband system temperature of about $T_{\mathrm{SYS}} \approx 4.0 \times 10^{5} \mathrm{~K}$, which implies a sky transmission of about $3 \%$ at zenith.

All planets suitable for determining the telescope efficiency were too low on sky at the time of the observations

Table 1. Summary of terahertz CO observations. The position (1950) of Orion BN/KL is $5^{\mathrm{h}} 32^{\mathrm{m}} 47^{\mathrm{s}}$ RA and $-5^{\circ} 24^{\prime} 23^{\prime \prime}$ Dec; NGC 2024 is $5^{\mathrm{h}} 39^{\mathrm{m}} 14^{\mathrm{s}} \mathrm{RA},-1^{\circ} 55^{\prime} 59^{\prime \prime}$ Dec; and W $3(\mathrm{OH})$ is $2^{\mathrm{h}} 23^{\mathrm{m}} 17^{\mathrm{s}} \mathrm{RA}$ and $+61^{\circ} 38^{\prime} 57^{\prime \prime}$ Dec. For Orion BN/KL, the lettering (a-f) in the region column corresponds to the panels in Fig. 1. $v_{\text {peak }}$ is the centroid velocity from a Gaussian fit.

\begin{tabular}{cccccc}
\hline \hline $\begin{array}{l}\text { Region } \\
(\text { Offset }) \\
\left(\Delta \alpha^{\prime \prime}, \Delta \delta^{\prime \prime}\right)\end{array}$ & $\begin{array}{c}\text { integ. } \\
\text { time } \\
\text { min. }\end{array}$ & $\begin{array}{c}\text { peak } \\
T_{\mathrm{A}}^{*}\end{array}$ & $\begin{array}{c}v_{\text {peak }} \\
\mathrm{km} \mathrm{s}^{-1}\end{array}$ & $\begin{array}{c}\Delta v \\
\mathrm{~km} \mathrm{~s}^{-1}\end{array}$ & $\begin{array}{c}\int T_{\mathrm{A}}^{*} \mathrm{~d} v \\
\mathrm{~K} \mathrm{~km} \mathrm{~s}^{-1}\end{array}$ \\
\hline Orion BN/KL & & & & & \\
"a" (+25, +200) & 4.2 & $\ldots$ & $\ldots$ & $\ldots$ & $\ldots$ \\
"b" (+25, +140) & 2.1 & 33 & 9.8 & 5.1 & 86 \\
"c" $(0,+60)$ & 4.2 & 49 & 11.0 & 20.9 & 1100 \\
"d" $(0,0)$ & 6.3 & 50.3 & 6.1 & 40.0 & 2200 \\
"e" $(0,-45)$ & 4.2 & 65.5 & 7.6 & 7.3 & 1500 \\
"f" $(0,-90)$ & 4.2 & 58 & 7.2 & 8.5 & 530 \\
NGC 2024 & 6.3 & $\ldots .$. & $\ldots .$. & $\ldots .$. & $\ldots .$. \\
W 3(OH) & 8.6 & 9 & -47 & 11 & 100 \\
\hline
\end{tabular}

to permit any measurements. With the same receiver tuned to $0.81 \mathrm{THz}$, we measured a main-beam efficiency of about $41 \%$ (Kawamura et al. 1999b). Fitting the efficiencies of all the receivers on the HHT to the Ruze formula, we expect a main beam efficiency of $19 \pm 5 \%$ near $1 \mathrm{THz}$. Simply extrapolating forward coupling efficiencies measured at the HHT at lower frequencies (Wilson et al. 2001) to $1.036 \mathrm{THz}$, we get an efficiency on the Moon of about $60 \%$, which is an upper limit to the coupling efficiency to our source. Since the sources are somewhat extended in $\mathrm{CO}(J=9 \rightarrow 8)$, the actual coupling efficiency lies at some intermediate value between $19 \%$ and $60 \%$. We take $40 \%$ for the source coupling efficiency with a conservative uncertainty of about $25 \%$. At the level of our sensitivity, we do not expect the error beam contribution in the spectra to be significant. Scaling the beam size we measured near $800 \mathrm{GHz}$, we expect it to be about 8.5" FWHM at $1.036 \mathrm{THz}$. The pointing model was previously established by observations of $\mathrm{CO}(J=7 \rightarrow 6)$ with the same receiver, and the pointing accuracy is about $\pm 3^{\prime \prime}$.

A $\log$ of our observations is given in Table 1 . We observed a position toward $\mathrm{BN} / \mathrm{KL}$ and 6 offsets to the $\mathrm{N}$ and $\mathrm{S}$ along a ridge centered roughly on $\mathrm{BN} / \mathrm{KL}$. These are all in OMC-1. We also observed two other regions, W $3(\mathrm{OH})$ and NGC 2024. Emission was detected in OMC-1 and tentatively from $\mathrm{W} 3(\mathrm{OH})$, but not toward NGC 2024. The spectra taken in Orion are shown in Fig. 1 along side a SHARC $350 \mu$ m continuum image (Lis et al. 1998) indicating where the spectra were taken.

\section{Results and discussion}

Line emission from the $\mathrm{CO}(J=9 \rightarrow 8)$ transition was detected with approximately the same peak intensity, $T_{\mathrm{A}}^{*} \sim 50 \mathrm{~K}$, at 5 pointings extending about $4^{\prime} \mathrm{N}-\mathrm{S}$ along the ridge, centered near BN/KL. This antenna temperature is similar to that measured by a KAO instrument (Röser et al. 1991), which has a source coupling efficiency roughly similar to our receiver. 
There are several spurious features apparent in the spectra in Fig. 1: a peak near $v \sim 60 \mathrm{~km} \mathrm{~s}^{-1}$ in panel a, and components near $\sim 120 \mathrm{~km} \mathrm{~s}^{-1}$ in panels $\mathrm{c}$ and $\mathrm{d}$. These features were identified as spurious because they appeared as narrow strong spikes in only one of the scans that were averaged. The BN/KL region itself exhibits very broadened line emission with a peak antenna temperature of $T_{\mathrm{A}}^{*}=50 \mathrm{~K}$, and the line is well-fitted by a single Gaussian component whose $F W H M$ line width is $40 \mathrm{~km} \mathrm{~s}^{-1}$ centered at $v_{\mathrm{LSR}}=+6.1 \mathrm{~km} \mathrm{~s}^{-1}$. The uncertainty in the velocity is approximately $1 \mathrm{~km} \mathrm{~s}^{-1}$. Elsewhere, the spectra are composed of much narrower components. At 2.3' north of IRc2, the line emission occurs at a single velocity component near $v_{\mathrm{LSR}} \approx 10 \mathrm{~km} \mathrm{~s}^{-1}$. At $1^{\prime}$ north of IRc2, the spectrum peaks at $11 \mathrm{~km} \mathrm{~s}^{-1}$, but has a noticeable asymmetric profile with a pronounced blue-shifted emission wing. At the midpoint between Orion-S and KL, $0.75^{\prime}$ south of IRc2, there are three distinct components, two near $6 \mathrm{~km} \mathrm{~s}^{-1}$ and $10 \mathrm{~km} \mathrm{~s}^{-1}$, and tentatively another at $v_{\mathrm{LSR}} \approx 19 \mathrm{~km} \mathrm{~s}^{-1}$. At the position of Orion-S, $1.5^{\prime}$ south of $\mathrm{KL}$, there is a component at $6 \mathrm{~km} \mathrm{~s}^{-1}$ and another at $9 \mathrm{~km} \mathrm{~s}^{-1}$. Line emission was not detected $3.3^{\prime} \mathrm{N}$ of KL, where the $1-\sigma$ upper limit to the integrated line strength is $\sim 30 \mathrm{~K} \mathrm{~km} \mathrm{~s}^{-1}$.

We can immediately make several general suppositions regarding the physical conditions along the central $\sim 4^{\prime}$ region near KL/IRc2. First, the peak antenna temperature is fairly constant within this extended region, with $T_{\mathrm{A}}^{*} \approx 50 \mathrm{~K}$. Since the $\mathrm{CO}(J=7 \rightarrow 6)$ line towards Orion $\mathrm{KL}$, which was observed with the same receiver, has almost exactly the same peak brightness temperature (Kawamura et al. 1999a), the line center is most certainly optically thick. In their survey of giant molecular clouds, Boreiko \& Betz (1991) determined that the line emission of ${ }^{12} \mathrm{CO}(J=9 \rightarrow 8)$ is optically thick in all cases, and we would expect Orion-KL to be similar to these, in contrast to earlier work by Goldsmith et al. (1981) and Schultz et al. (1985) who claimed that lower- $J$ CO lines at line center were optically thin towards Orion-KL. Since the line is optically thick, the line brightness temperature can be used to compute the excitation temperature of the gas, $T_{\mathrm{ex}}=154 \pm 35 \mathrm{~K}$. This value is consistent with many other independent measurements of the gas temperature near the plateau/core region made using high-density molecular tracers (Boreiko et al. 1989; Schloerb et al. 1983; Blake et al. 1987). The critical density of the $\operatorname{CO}(J=9 \rightarrow 8)$ transition at these temperatures is $n \approx 10^{6} \mathrm{~cm}^{-3}$. Since the emission appears to be very strong everywhere along the ridge, we conclude that the gas responsible for the emission has a minimum density of $n \approx 10^{6} \mathrm{~cm}^{-3}$ with gas temperatures near $T_{\text {kin }} \approx 150 \mathrm{~K}$. As might be expected, the size of the region traced by $\mathrm{CO}(9 \rightarrow 8)$ is similar to that measured using $\mathrm{CO}(7 \rightarrow 6)$ (Schmid-Burgk et al. 1989), although it appears somewhat larger than those measured with higher- $J$ lines of $\mathrm{CO}$, which showed that the emission extends to $\sim 1^{\prime}$ at the $J=17 \rightarrow 16$ line (Boreiko et al. 1989) and about 1.5' $F W H M$ in $J=21 \rightarrow 20$ (Storey et al. 1981). The fact that the line emission is thus extended allows us to identify the material as belonging to either the warm components in the ridge or to photon dominated regions.

We identify three physical components in the spectra, distinguished by their Doppler velocities and spectral profile. The large linewidth at the position of Orion KL marks the site of the powerful molecular outflow located here (Zuckerman et al. 1976; Kwan \& Scoville 1976). Previous observations have shown that the molecular outflow, which appears to originate near the source IRc2, is spatially very compact, weakly bipolar, and extends only $\sim 30^{\prime \prime}$ across in CO $(J=3 \rightarrow 2)$ (Erickson et al. 1982) and about $10^{\prime \prime}$ as traced by $\mathrm{SiO}(v=$ $0, J=2 \rightarrow 1$ ) (Wright et al. 1983). We do not detect any component $|V|>40 \mathrm{~km} \mathrm{~s}^{-1}$ in our spectra, but we may be limited by sensitivity. The minimum beam-averaged $\mathrm{CO}$ column density towards IRc2 is $4 \times 10^{16} \mathrm{~cm}^{-2}$, assuming that the gas is thermalized at $T \approx 154 \mathrm{~K}$. Away from KL/IRc2, there are two quiescent components in the spectra. We see a dominant feature at $v_{\mathrm{LSR}} \approx 10 \mathrm{~km} \mathrm{~s}^{-1}$ to the north, and another at $v_{\mathrm{LSR}} \approx 6 \mathrm{~km} \mathrm{~s}^{-1}$ appears to become more prominent towards the south of KL.

Ho \& Barrett (1978) observed $\mathrm{NH}_{3}$ towards the BN/KL core and detected blended line emission from two apparently distinct kinematic components, and interpreted this as arising from two clouds along the line of sight with $v_{\text {LSR }}$ differing by about $2 \mathrm{~km} \mathrm{~s}^{-1}$. They further speculated that the clouds were colliding and that this collision was somehow triggering the energetic events in the core region. In their study of the core region of Orion, Boreiko et al. (1989) also identified two blended velocity components in their $\mathrm{CO}(J=17 \rightarrow 16)$ spectra, which they called the "wide-" and "narrow-" components, at $v_{\mathrm{LSR}} \approx 6-8 \mathrm{~km} \mathrm{~s}^{-1}$ and $10-11 \mathrm{~km} \mathrm{~s}^{-1}$, respectively, in good agreement with those seen by Ho \& Barrett (1978). However, both observations have low angular resolution and could not unambiguously determine whether the lines originated from the same spatial location, or were simply emission from different regions but blended by their instrument. Boreiko et al. (1989) also did not examine the issue of the kinematic relationship between these cloud components. Several cloud components are also seen by Boreiko et al. (1988) who observed the $\mathrm{C}_{\text {II }}{ }^{2} \mathrm{P}_{3 / 2} \rightarrow{ }^{2} \mathrm{P}_{1 / 2}$ fine-structure line towards the Orion core.

Womack et al. (1991) observed the ground-state rotational transition of $\mathrm{N}_{2} \mathrm{H}^{+}$during a survey and discovered that it was depleted in the most energetic core region, but could be detected from the quiescent material. Using this tracer they were able to filter out the wide line emission in the outflow, which tends to dominate the spectra taken with instruments with low angular resolution. Their observations indicated that there are two quiescent cloud components, at $v_{\mathrm{LSR}} \sim 9.5 \mathrm{~km} \mathrm{~s}^{-1}$ and $7.2 \mathrm{~km} \mathrm{~s}^{-1}$, which are spatially separated for the most part, but which neatly overlap along the line of sight towards the BN/KL region.

Wang et al. (1993) exploited this technique further by observing methyl acetylene $\left(\mathrm{CH}_{3} \mathrm{C}_{2} \mathrm{H}\right)$, a molecule that apparently behaves in a way chemically similar to $\mathrm{N}_{2} \mathrm{H}^{+}$. With the angular resolution of the map improved by a factor of better than 2, they were able to largely confirm the conclusions of Womack et al. (1991). However, Wang et al. (1993) detected three spatially distinct velocity components and that the two that overlap near BN/KL have velocities of $v_{\mathrm{LSR}} \sim 8 \mathrm{~km} \mathrm{~s}^{-1}$ and $\sim 10 \mathrm{~km} \mathrm{~s}^{-1}$. The cloud detected furthest south has a velocity of $\sim 6.5 \mathrm{~km} \mathrm{~s}^{-1}$. The $8 \mathrm{~km} \mathrm{~s}^{-1}$ velocity component detected by Wang et al. (1993) is apparently detected at only one position by Womack et al. (1991), and is not seen in the region 
by other methods. This may indicate a possible complication in using a tracer whose chemistry in the interstellar medium is at best uncertain, especially in a region of vigorous activity.

Our new CO data with superior angular resolution supports the model that there are 2 quiescent clouds in the Orion ridge, and that they overlap or intersect in the vicinity of BN/KL. That the overlap is coincident within about $20^{\prime \prime}$ of the place of extreme activity in the Orion core is suggestive of a causal relation, as was postulated by Ho \& Barrett (1978). The fact that there appears to be line emission at an intermediate velocity of the two clouds, as evidenced by the $\mathrm{N}_{2} \mathrm{H}^{+}$and $\mathrm{CH}_{3} \mathrm{C}_{2} \mathrm{H}$ observations, further suggests that there is activity between or at the interface of the two clouds. In any case, the evidence unequivocally rules out the possibility that the observed velocity gradient along the ridge stems from a rotation of the inner ridge.

We briefly turn our attention to the asymmetry in the line profile in the spectrum taken $60^{\prime \prime} \mathrm{N}$ of IRc2. We have explicitly favored that the cause of the blue wing is a second component at a lower velocity. However, at $60^{\prime \prime} \mathrm{N}$ of KL, we are about $20^{\prime \prime} \mathrm{N}$ of a peak in $\mathrm{H}_{2}$ vibrational emission detected by Beckwith et al. (1978). We do not believe that the emission in the blue wing in the $\mathrm{CO}$ spectrum taken $\mathrm{N}$ of $\mathrm{KL}$ originates in a shock front causing the $\mathrm{H}_{2}$ emission. This is because of the fact that even though an extended blue wing is seen in the $\mathrm{H}_{2}$ vibrational spectrum (Nadeau et al. 1982), the line profile in $\mathrm{CO}(9 \rightarrow 8)$ is simply not as wide. Also, the fact that we see the same velocity component at other positions leads us to prefer a simple two-component quiescent gas model, as favored by Boreiko et al. (1989).

To conclude, our experiment convincingly demonstrates the feasibility of conducting observations from a ground-based site at $\mathrm{THz}$ frequencies, especially in the context of heterodynemode observing. FTS radiometer measurements at and near the ALMA site (Matsushita et al. 1999; Paine et al. 2000) as well as Mauna Kea (Pardo et al. 2001) show that there are several potentially useful windows between $1.0 \mathrm{THz}$ and $1.5 \mathrm{THz}$, where the transmission reaches $\sim 35 \%$, and that such conditions occur reasonably frequently. Efforts are currently underway to measure the sky characteristics at a nearby site with yet higher elevation (Paine \& Blundell, private communication). This introduces the exciting prospect of developing a site for a $\mathrm{THz}$ telescope.

This work is based in part on measurements made with the Heinrich Hertz Telescope, which is operated by the Submillimeter Telescope Observatory on behalf of Steward Observatory and the Max-Planck-Institut für Radioastronomie.

\section{References}

Baars, J. W. M., Martin, R. N., Mangum, J. G., McMullin, J. P., \& Peters, W. L. 1999, PASP, 111, 627
Beckwith, S., Persson, S. E., Neugebauer, G., \& Becklin, E. E. 1978, ApJ, 223, 464

Blake, G. A., Sutton, E. C., Masson, C. R., \& Phillips, T. G. 1987, ApJ, 315, 621

Boreiko, R. T., Betz, A. L., \& Zmuidzinas, J. 1988, ApJ, 325, L47

Boreiko, R. T., Betz, A. L., \& Zmuidzinas, J. 1989, ApJ, 337, 332

Boreiko, R. T., \& Betz, A. L. 1991, ApJ, 369, 382

Erickson, N. R., Goldsmith, P. F., Snell, R. L., et al. 1982, ApJ, 261, L103

Genzel, R., \& Stutzki, J. 1989, ARA\&A, 27, 41

Goldsmith, P., Erickson, N. R., Fetterman, H. R., et al. 1981, ApJ, 243, L79

Hasegawa, T., Kaifu, N., Inatani, J., et al. 1984, ApJ, 283, 117

Ho, P. T. P., \& Barrett, A. H. 1978, ApJ, 224, L23

Kawamura, J., Tong, C.-Y. E., Blundell, R., et al. 1999a, IEEE Trans. Appl. Supercond., 9, 3753

Kawamura, J., Hunter, T. R., Tong, C.-Y. E., et al. 1999b, PASP, 111, 1088

Kawamura, J., Blundell, R., Tong, C.-Y. E., et al. 2000, IEEE Trans. MTT, 48, 683

Kutner, M. L., Evans, N. J., \& Tucker, K. D. 1976, ApJ, 209, 452

Kutner, M. L., Tucker, K. D., Chin, G., \& Thaddeus, P. 1977, ApJ, 215,521

Kwan, J., \& Scoville, N. Z. 1976, ApJ, 210, L39

Lis, D. C., Serabyn, E., Keene, J., et al. 1998, ApJ, 509, 299

Matsushita, S., Matsuo, H., Pardo, J., \& Radford, S. J. E. 1999, PASJ, 51,603

Menten, K., \& Reid, M. J. 1995, ApJ, 445, L157

Nadeau, D., Geballe, T. R., \& Neugebauer, G. 1982, ApJ, 253, 154

Paine, S., Blundell, R., Papa, D. C., Barrett, J. W., \& Radford, S. J. E. 2000, PASP, 112, 108

Pardo, J. R., Serabyn, E., \& Cernicharo, J. J. 2001, Quant. Spectrosc. Radiat. Transfer, 68, 419

Röser, H.-P. 1991, Infrared Phys., 32, 385

Schloerb, F. P., Friberg, P., Hjalmarson, A., Höglund, B., \& Irvine, W. M. 1983, ApJ, 264, 161

Schmid-Burgk, J., Densing, R., Krugel, E., et al. 1989, A\&A, 215, 150

Schultz, G. V., Durwen, E. J., Röser, H. P., Wattenbach, R., \& Sherwood, W. A. 1985, ApJ, 291, L59

Storey, J. W. V., Watson, D. M., Townes, C. H., Haller, E. E., \& Hansen, W. L. 1981, ApJ, 247, 136

Tong, C.-Y. E., Blundell, R., Papa, D. C., et al. 1999, IEEE Microwave Guided Wave Lett., 9, 366

Vogel, S. N., Bieging, J. H., Plambeck, R. L., Welch, W. J., \& Wright, M. C. H. 1985, ApJ, 296, 600

Wang, T. Y., Wouterloot, J. G. A., \& Wilson, T. L. 1993, A\&A, 277, 205

Wilson, T. L., Serabyn, E., \& Henkel, C. 1986, A\&A, 167, L17

Wilson, T. L., et al. 2001, Science with Atacama Large Millimeter Array, ed. A. Wooten., ASP Conf. Ser., 235, 257

Womack, M., Ziurys, L. M., \& Wyckoff, S. 1991, ApJ, 370, L99

Wright, M. C. H., Plambeck, R. L., Vogel, S. N., Ho, P. T. P., \& Welch, W. J. 1983, ApJ, 267, 41

Zuckerman, B., Kuiper, T. H. H., \& Rodriguez-Kuiper, E. N. 1976, ApJ, 209, L137 